\title{
The 2005 October Multiwavelength Campaign of GRS 1915+105
}

\author{
Y. Ueda ${ }^{* a}$, R. Ishioka ${ }^{b}$, K. Sekiguchi $^{b}$, M. Ribo $^{c}$, J. Rodriguez $^{d}$, S. Chaty ${ }^{d, e}$, \\ J. Greiner ${ }^{f}$, G. Sala ${ }^{f}$, Y. Fuchs ${ }^{d}$, P. Goldoni ${ }^{g}, d$, S. Covino ${ }^{h}$, G.G. Pooley ${ }^{i}$, \\ P. Edwards ${ }^{j}$, A. Tzioumis ${ }^{k}$, H. Lehto ${ }^{l}$, E. Gerard ${ }^{m}$, P. Colom ${ }^{m}$, J. Martin ${ }^{m}$,
} S.A. Trushkin ${ }^{n}$, A.J. Castro-Tirado ${ }^{o}$, D. Hannikainen ${ }^{p}$, H. Sudo ${ }^{q}$, M. Honma ${ }^{r}$, F. Iwamuro ${ }^{a}$, K. Kubuta ${ }^{a}$, K. Yamaoka ${ }^{s}$, C. Done ${ }^{t}$, S. Naik ${ }^{u}$, Y. Fukazawa ${ }^{v}$, L. Angelini ${ }^{w}$, H. Awaki ${ }^{x}$, K. Ebisawa ${ }^{u}$, K. Iwasawa ${ }^{f}$, N. Kawai ${ }^{y}$, K. Kinugasa ${ }^{z}$, M. Kokubun ${ }^{a a}$, T. Kotani ${ }^{y}$, A. Kubota ${ }^{a b}$, T. Murakami ${ }^{a c}$, M. Namiki ${ }^{a d}$, H. Takahashi ${ }^{v}$, T. Yaqoob ${ }^{a e}$, D. Yonetoku ${ }^{a c}$, and A. Yoshida ${ }^{s}$

${ }^{a}$ Kyoto University, Japan ${ }^{b}$ Subaru Telescope/NAOJ, Japan

${ }^{c}$ Universitat de Barcelona, Spain dCEA-Saclay, France

${ }^{e}$ Université Paris VII, France ${ }^{f}$ Max-Planck-Institut für extraterrestrische Physik, Germany

${ }^{g}$ APC/UMR 7164, France ${ }^{h}$ INAF-OAB, Italy

${ }^{i}$ University of Cambridge, UK ${ }^{j}$ CSIRO, Australia

${ }^{k}$ ATNF, Australia ${ }^{l}$ University of Turku, Finland

${ }^{m}$ Observatoire de Meudon, France ${ }^{n} S A O$, Russia

${ }^{o} I A A$, Spain ${ }^{p}$ University of Helsinki, Finland

${ }^{q}$ Gifu University, Japan ${ }^{r}$ Mizusawa VERA Observatory/NAOJ, Japan

${ }^{s}$ Aoyama Gakuin University, Japan ${ }^{t}$ University of Durham, UK

"ISAS/JAXA, Japan ${ }^{v}$ Hiroshima University, Japan

${ }^{w}$ GSFC/NASA, USA ${ }^{x}$ Ehime University, Japan

${ }^{y}$ Tokyo Institute of Technology, Japan ${ }^{z}$ Gunma Astronomical Observatory, Japan

${ }^{a a}$ University of Tokyo, Japan ${ }^{a b}$ RIKEN, Japan

${ }^{a c}$ Kanazawa University, Japan ad Osaka University, Japan

ae Johns Hopkins University, USA

E-mail: ueda@kusastro.kyoto-u.ac.jp

We report the first results of a large multiwavelength campaign of GRS 1915+105 performed between 2005 October 16 to October 18 with Suzaku, INTEGRAL, RXTE, Swift, Spitzer, and ground observatories including Subaru, ESO/NTT, REM (infrared), VLA, ATCA, Nancay, Ryle, and RATAN (radio). GRS $1915+105$ was firstly in the stable state with a $6 \mathrm{~Hz}$ QPO but then entered into the oscillation phase identified as Class $\theta$. The Suzaku spectra in the $1-80 \mathrm{keV}$ band are very soft, approximated by a photo index of 3-3.8 with varying iron-K features in different Xray states. The simultaneous Subaru and X-ray light curves in the oscillation phase indicate that infrared flares occur after the X-ray dip with variable time delay. This result strongly suggests that the disc transition from State $\mathrm{C}$ to State A triggers the ejection of jet, which eventually produces infrared/radio flares by internal shock.

VI Microquasar Workshop: Microquasars and Beyond

September 18-22 2006

Società del Casino, Como, Italy

*Speaker. 


\section{Introduction}

Microquasars provide us with an ideal laboratory to study the origin of a relativistic jet and the relation with accretion onto a black hole [1]. Multiwavelength studies of these objects are of particular importance since high energy radiation (X-ray to gamma-ray) traces the accretion flow in the vicinity of a black hole while the jet emitting synchrotron radiation can be observed in the radio to near infrared band.

GRS $1915+105$ is the most important, archetype microquasar, which has been persistently active since its discovery in 1992 (for a recent review see [2]). In X rays, it often shows dramatic temporal and spectral variations in quasi-regular cycles, most probably due to the rapid transition of the accretion disc state. GRS 1915+105 also exhibits various classes of jet activity, which is closely coupled with the disc state. Although many multiwavelength observations of this source have been performed so far, the physics of the jet formation is far from being fully understood because of an apparent large diversity of phenomena. To establish a unified picture of the disc-jet connection, it is crucial to take more complete sets of high quality multiwavelength data covering the radio, infrared, and X-ray bands.

Suzaku [3], the 5th Japanese X-ray satellite launched on 2005 July 10, observed GRS 1915+105 on 2005 October 16-18 (UT throughout the paper) as a part of the science working group's observation program. Taking this opportunity, we have coordinated a large multiwavelength campaign involving other space and ground observatories, based on our experience of the previous, then "largest" campaign performed with ASCA in 2000 April [4]. In this paper, we report preliminary results obtained from these observations. More detailed results shall be published in forthcoming papers.

\section{Observations and Data Reduction}

\subsection{Summary}

The following satellites and ground facilities participated in the 2005 October multiwavelength campaign of GRS 1915+105 (main period: October 16-18); (from space) Suzaku (covering 0.2$600 \mathrm{keV}$ with the XIS and HXD instruments, PI: Ueda), INTEGRAL (3 keV-100 keV with JEM-X and ISGRI, PI: Rodriguez), Swift (1-200 keV with XRT and BAT, PI: Greiner), RXTE (2-200 keV with PCA and HEXTE, PI: Morgan), Spitzer (5.2-38 $\mu \mathrm{m}$, PI: Fuchs), (from ground) Subaru ( $K^{\prime}, \mathrm{PI}$ : Sekiguchi and Ishioka), ESO/NTT ( $K s$, PI: Chaty), REM ( $K$, PI: Goldoni), VLA (1.4, 5, 8.5, and 15 GHz, PI: Ribo), ATCA (4.86 and 8.46 GHz, PI: Edwards), Ryle Telescope (15 GHz, PI: Pooley), Nancay Telescope (1.4 and $2.7 \mathrm{GHz}$, PI: Lehto), and RATAN (1.0-11.2 GHz, PI: Trushkin) ${ }^{1}$.

\subsection{Suzaku Observations}

Suzaku observed GRS 1915+105 from 2005 Oct 16 16:42 to Oct 18 23:16 for a net exposure of $\approx 80 \mathrm{ksec}$. Suzaku carries four sets of X-ray mirrors each with a focal plane X-ray CCD camera, the

\footnotetext{
${ }^{1}$ We also coordinated near infrared observations at the Calar Alto observatory in Spain (PI: Castro-Tirado), Infrared Survey Facility in South Africa, Gunma Astronomical Observatory in Japan, and the ISAS infrared telescope in Japan, which were unsuccessful due to bad weather. In radio we performed observations with e-VLBI (PI: Sudoh) and VERA (PI: Honma) but the source flux was below the detection limits.
} 
X-ray Imaging Spectrometer (XIS-0, XIS-1, XIS-2, and XIS-3 [5]), and a non-imaging instrument called the Hard X-ray Detector (HXD [6]), which consists of the Si PIN photo-diodes and GSO scintillation counters. The XIS, PIN, and GSO simultaneously covers the energy band of 0.2-12 $\mathrm{keV}, 10-60 \mathrm{keV}$, and 40-600 keV, respectively. In this paper we only present the results of the XIS and PIN since very careful analysis is required for the GSO, whose calibration is still in progress. To minimize pile-up (double photon events) of the XIS data due to the extreme brightness of this source, XIS-0, XIS-2, and XIS-3 were operated with the 1/8 window option, which reduced the exposure time per frame from $8 \mathrm{sec}$ to $1 \mathrm{sec}$. XIS- 1 was operated with the $0.1 \mathrm{sec}$ burst plus $1 / 8$ window option, although the 1/8 window option had to be turned off for XIS-1 before Oct 18 00:26 due to an operational error. We use the XIS-1 events to derive unsaturated light curves with a $1 \mathrm{sec}$ time resolution (after Oct 18 00:26), while we use XIS-0, 2 and 3 data to make the spectra with high photon statistics.

\subsection{Subaru Observations}

Near infrared $\left(K^{\prime}\right)$ photometric observations of GRS 1915+105 were conducted using the CISCO instrument [7] on the 8.2-m Subaru telescope on 2005 October 17 05:09 to 06:42 and October 18 05:34 to 08:45. We performed $K^{\prime}$-band photometry in the high-speed imaging mode. In this mode, a sequence of dataset includes 96 object images with a size of $13.5^{\prime \prime} \times 13.5^{\prime \prime}$ and a deadtime of $0.09 \mathrm{sec}$ between each image. We set the exposure time $1 \mathrm{sec}$. The telescope was dithered at four positions with offsets of $1.2^{\prime \prime}$ and $0.5^{\prime \prime}$ for RA and Dec, respectively, around the centre of $19^{\mathrm{h}} 15^{\mathrm{m}} 11.1^{\mathrm{s}}$ and $10^{\circ} 56^{\prime} 44.5^{\prime \prime}$. The typical deadtime between each sequence used for the data recording and telescope pointing was about $50 \mathrm{sec}$.

The data were reduced by using the MSCRED and DAOPHOT packages of IRAF ${ }^{2}$. After dark subtracting and flat-fielding by the standard $K^{\prime}$ flat frame, we masked the star positions for each image and normalized the background levels. Then we created a median sky frame and subtracted it from the object images. We finally performed PSF photometry, and made differential light curves using 'Star A' in [8] as a comparison star.

\subsection{REM Observations}

The REM robotic telescope [9] observed GRS 1915+105 during the nights of 2005 October 14, 15, 16, 17 and 18 at La Silla, Chile. The telescope is a fully-robotic $60 \mathrm{~cm}$ telescope equipped with the REMIR Rockwell Infrared Camera $\left(512 \times 512\right.$ pixels). The field of view is $9.9^{\prime} \times 9.9^{\prime}$ and the plate scale is $1.2^{\prime \prime}$ pixel $^{-1}$.

The observations lasted from a minimum of 8 minutes to a maximum of 90 minutes on October 18 due to unfavorable visibility constraints. On each night, frames in the $K$ band were acquired with integration time of $150 \mathrm{sec}$; in each band five frames with integration time of $30 \mathrm{sec}$ were averaged. The condition were photometric on October 15, 16, 17 and 18, while on October 19 some clouds were present. The seeing was around $1.0^{\prime \prime}$ during all the observations. The $J H K$ filters are the one defined by the University of Hawaii (e.g., [10] and references therein). Photometry was performed using SExtractor [11], and absolute photometry was obtained using 30 2MASS stars in the field

\footnotetext{
${ }^{2}$ IRAF is distributed by the National Optical Astronomy Observatories, which is operated by the Association of Universities for Research in Astronomy, Inc. under cooperative agreement with the National Science Foundation
} 
of view. However, the software is not able to separate GRS 1915+105 from a nearby star ('Star A' in [8]) in our images, the photometric results are thus contaminated. To separate the two components, we analyzed an average frame for each night using the PSF-fitting technique, in DAOPHOT [12].

\section{Multiwavelengh Light Curves}

\subsection{Light Curves}

Figure 1 shows the long term X-ray light curve of GRS 1915+105 taken with the RXTE All Sky Monitor (ASM) in the $2-12 \mathrm{keV}$ band centered at our our observation campaign epoch. As noticed from this figure, we caught the source about 40 days after it entered into the active phase with strong $X$-ray variability from the quasi-stable state that lasted for $\approx 60$ days.

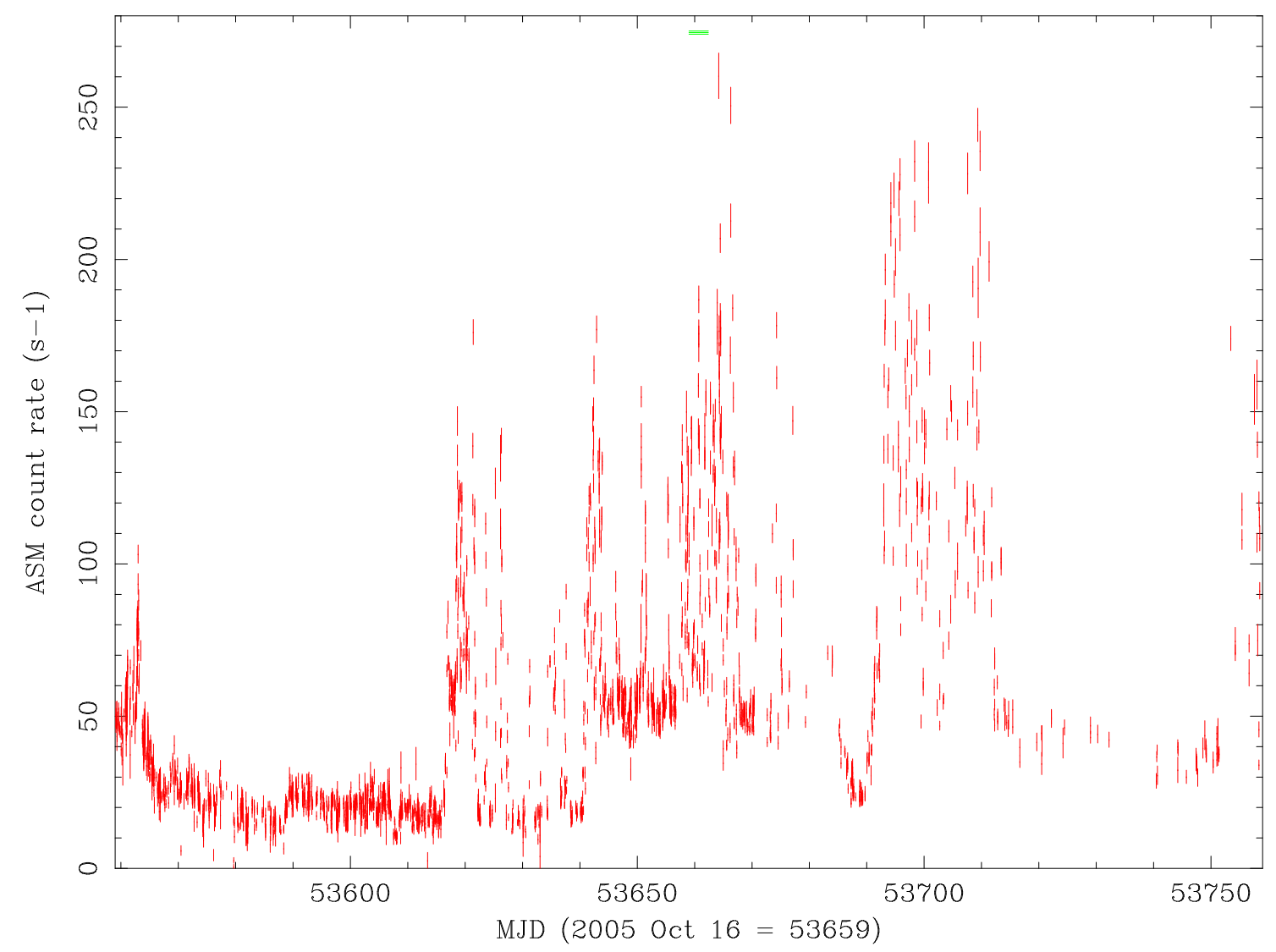

Figure 1: The RXTE ASM light curves of GRS 1915+105, centered at 2005 Oct 16.0. Our multiwavelengthcampaign epoch is denoted by the green bar below the top axis.

Figure 2 shows our multiwavelength light curves in the period from 2005 October 16 to 19 , sorted by the wavelength from the upper (longer) to the bottom. For convenience, hereafter we define $t$ as the time since 2005 October 16.0 in units of hours. It is seen that at $t \lesssim 40$ GRS $1915+105$ was in a X-ray stable state, except for a possible flare-like event seen at $t \simeq 21.5$. From $t \simeq 40$, the X-ray flux increased rapidly and entered into "oscillation" that lasted till $t \simeq 56$. From $t \simeq 66$ GRS $1915+105$ showed a similar X-ray flare (as most clearly seen in the continuous JEM-X 
light curve), followed by oscillation whose variability pattern is different from the previous one. Throughout the observation, the source was very bright in soft $X$ rays, $\approx 1-3$ Crab in the $2-10 \mathrm{keV}$ band corresponding to $(0.5-1.5) \times 10^{39} \mathrm{erg} \mathrm{s}^{-1}$ for a distance of $12.5 \mathrm{kpc}$. This is consistent with the fact, shown later, that the broad band spectra in the $1-80 \mathrm{keV}$ band are very soft, approximated by power law photon indices of 3-3.8.

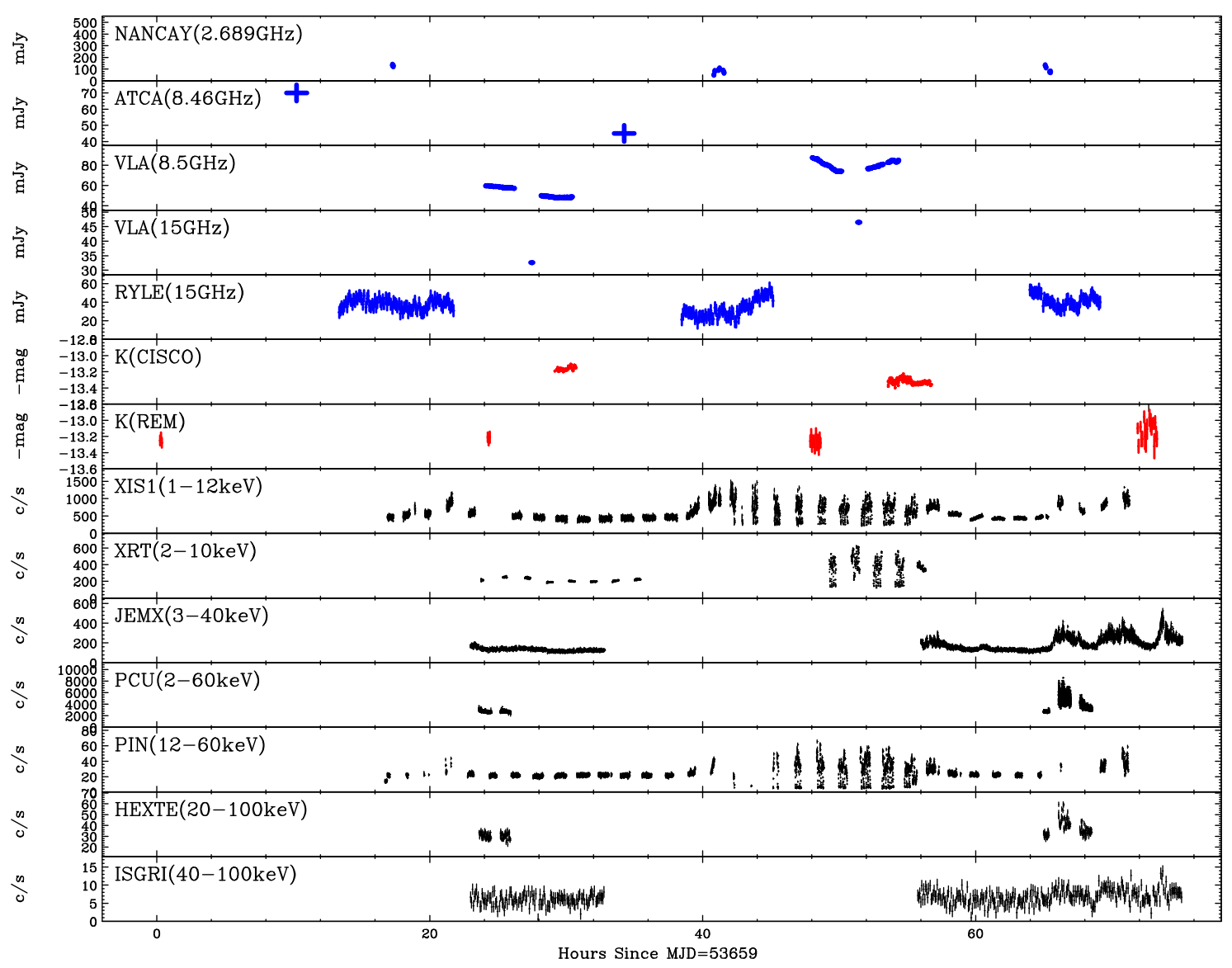

Figure 2: The multiwavelength light curves of GRS 1915+105.

The radio data indicate moderate jet activity during the campaign epoch. The $8.5 \mathrm{GHz}$ flux measured with the VLA was $\approx 50 \mathrm{mJy}$ on October 17 and $\approx 80 \mathrm{mJy}$ on October 18 , in the latter date showing a decay followed by a new flare. The multi-frequency VLA data show that the spectra were optically thin with an energy index of $\approx 0.8$ on both dates, evidence for synchrotron emitter ejected before the observation. The mean $K$ magnitudes is $13.0-13.4$, which is a typical or somewhat faint flux levels for this source. We found, however, evidence for significant short time variability with $<0.1$ mag levels from the Subaru data, which shall be presented in $\S 5$.

\subsection{X-ray States}

For comparison with previous studies of GRS $1915+105$ it is of the first interest to identify its X-ray "Class", or "State", according to the model-independent classification scheme by [13]. As 
seen from Figure 2, GRS 1915+105 continuously stayed in the "stable state" in the period of $t=$ $23-38$, which is similar to the plateau state [14], before entering into the oscillation phase. The soft $\mathrm{X}$-ray flux was much brighter than a typical value observed in the plateau state. To understand the nature of this state, we analyze the RXTE PCA data taken at the period of $t=23-26$. Figure 3(a) shows the X-ray color-color diagram, where the same definition of color as in [13] is adopted. Figure 3(b) shows the power spectral density in the total PCA band; a quasi periodic oscillation (QPO) at $\approx 6 \mathrm{~Hz}$ is detected. The presence of a low frequency QPO is the characteristics of State $\mathrm{C}$ (or Class $\chi$ if stable), although the X-ray colors (both $H R 1$ and $H R 2$ ) are much softer in our case than the typical valus often observed in State C. We therefore conclude that our "stable state" corresponds to State $\mathrm{C}$ having an extremely soft spectrum.
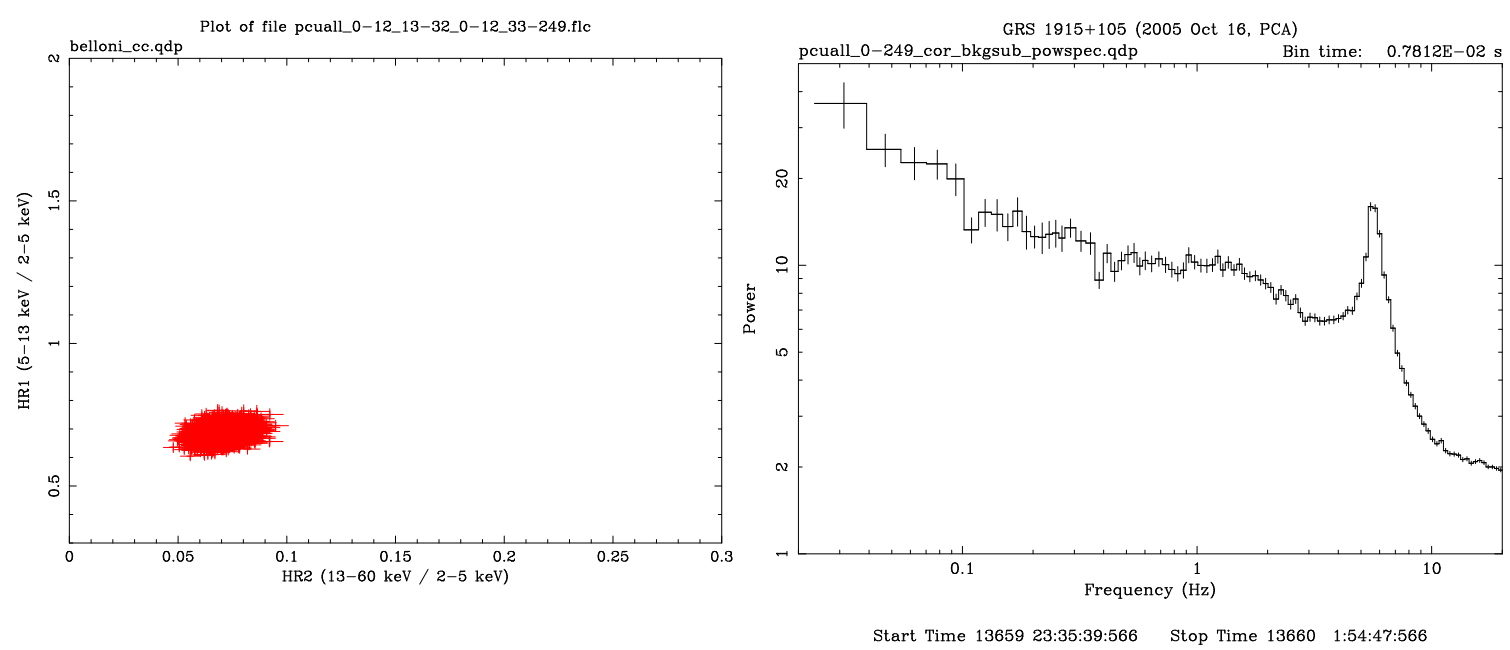

Figure 3: (Left) The X-ray color-color diagram from the RXTE PCA data taken at $t=23-26$. The color $H R 1$ and $H R 2$ is defined as the count-rate ratio between $5-13 \mathrm{keV}$ and $2-5 \mathrm{keV}$ and between $13-60 \mathrm{keV}$ and $2-5 \mathrm{keV}$, respectively. (Right) The power spectral density in the total PCA band (without background subtraction).

Figure 4 shows a blow-up of X-ray light curves $(t=48.4-48.9)$ in the oscillation phase taken with the Suzaku XIS-1 and PIN in the 1-12 keV and 12-60 keV band, respectively, together with the hardness ratio between them. We identify the X-ray variability pattern as Class $\theta$ in [13], where the transition occurs between State $\mathrm{C}$ and State A (soft dip) on a time scale of $\sim 10$ minutes.

\section{The Suzaku Spectra}

We analyze the broad band spectra of GRS $1915+105$ in the 1-80 keV band obtained with the Suzaku XIS and PIN in three different states, (a) "stable state", (b) "oscillation high" state, and (c) "oscillation low" state. The time region is taken between $t=25.6-38.6$ for (a) and $t=49.9-54.4$ for (b) and (c). The oscillation high and oscillation low state is defined as when the 12-60 keV PIN count rate is above $30 \mathrm{c} \mathrm{s}^{-1}$ and below $10 \mathrm{c} \mathrm{s}^{-1}$, respectively, within the above time region. We sum the spectra of XIS-0, 2 and 3, after excluding a core of the point spread function of the mirror to minimize the effect of pile-up. The absolute XIS flux is tuned to be smoothly connected with 


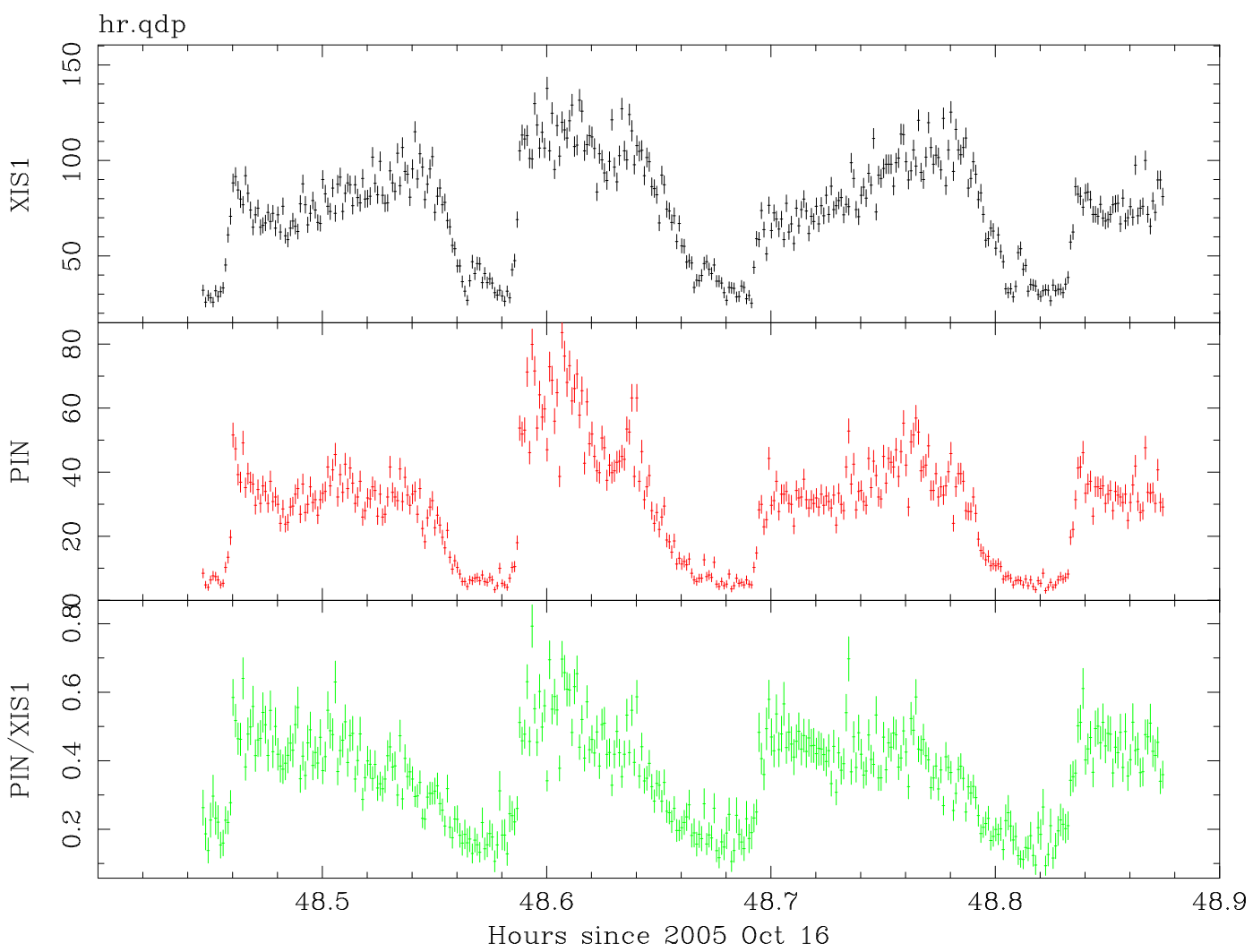

Figure 4: The X-ray light curves during the "oscillation" (class $\theta$ ). Upper: XIS-1 (1-12 keV), Middle: PIN (12-60 keV), Lower: hardness ratio (PIN/XIS-1).

the PIN flux. Model background spectra provided by the HXD team were subtracted from the PIN spectra.

The unfolded spectra were plotted in Figure 5. Here we employ a power law with the multicolor disc model [15] modified by an absorption with the solar abundances to approximate the continuum, although this simple model cannot provide acceptable fits particularly because of very complex features in the iron- $\mathrm{K}$ band. As noticed from the figure, while the broad band spectrum is softer in the oscillation low state (corresponding to State A in [13]) than in the other states (State C in [13]), the overall shape looks similar to one another.

The XIS spectra reveal that the iron-K features look different between the three states. In addition to a deep absorption-edge at $7.11 \mathrm{keV}$, discovered with the Chandra HETGS [16], we detect a narrow emission line at $\simeq 6.6 \mathrm{keV}$ in both stable and oscillation low states, whereas it is very weak in the oscillation high state. We also find a strong absorption line feature from $\mathrm{H}$ like iron ions at $\simeq 7.0 \mathrm{keV}$ in the oscillation low state, which is much weaker in the stable and oscillation high states. Here we do not discuss the presence of a broad iron line, for which very careful modelling of the continuum is required.

The iron- $\mathrm{K}$ emission line is evidence for the reflection of the continuum emission by moderately ionized matter. The reflector is most probably outer parts of the accretion disc, as constrained by its "narrow" line width. The absence of the iron-K narrow emission line in the oscillation high 
state implies that the inner part of the disc becomes geometrically thick in this accretion mode, which shields the Comptonizing plasma as seen from the outer disc.

Absorption lines of highly ionized ions indicate the presence of a disc wind in the line-of-sight between the continuum emitter and the observer. Recent observations show that the existence of a disc wind is quite common in high luminosity systems of an accreting compact object (e.g., [16] [17] [18]). The absence of the absorption-line in the stable state implies that the scale height of the disc wind is too small to cross the line-of-sight. In the oscillation high state, it is almost fully ionized by strong irradiation, making the absorption line of $\mathrm{H}$-like iron ions much weaker.

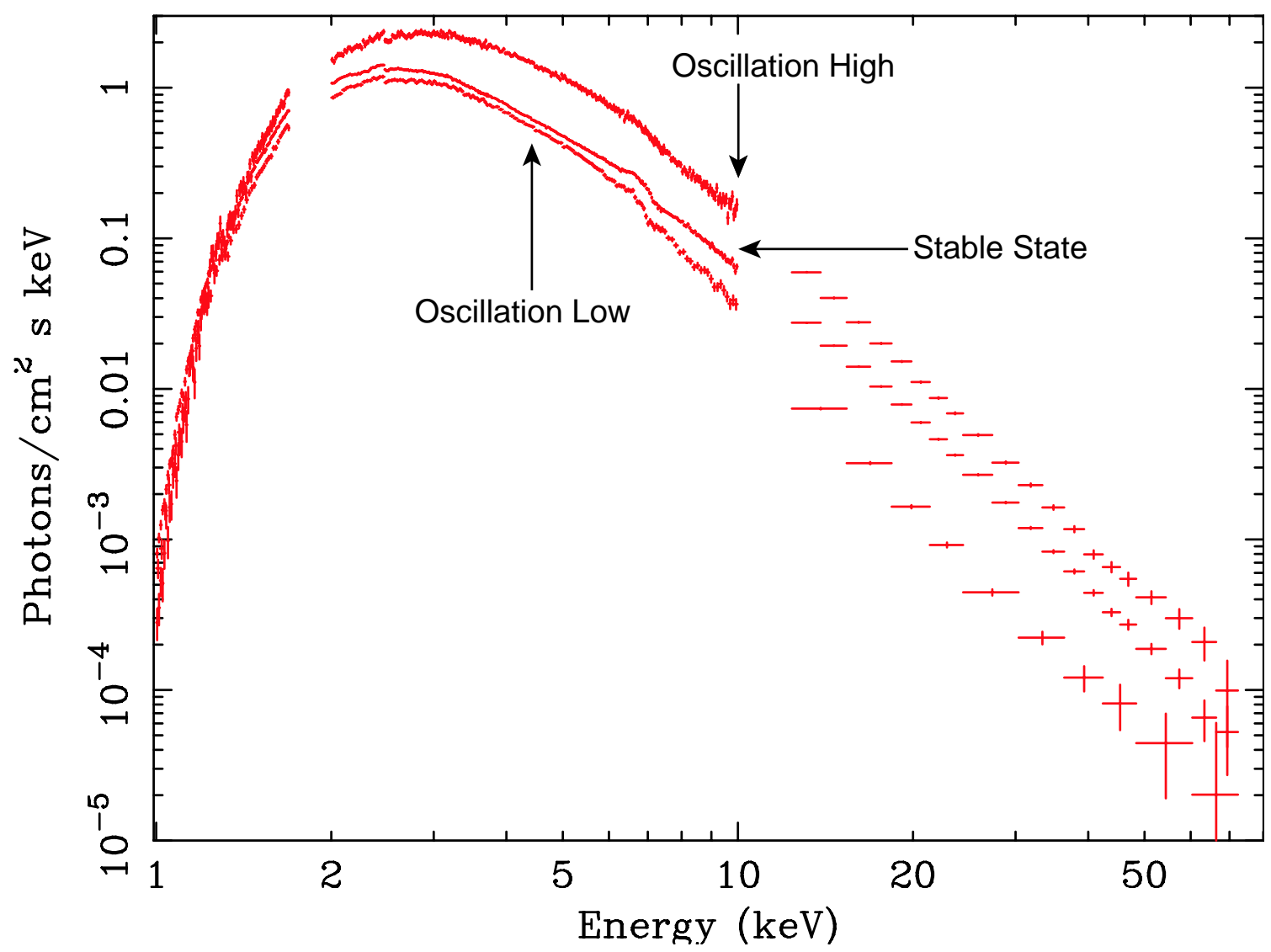

Figure 5: The Suzaku XIS+PIN spectra of GRS 1915+105 in the three different states (see text), unfolded for the detector response. The Si-K band $(1.7-2.0 \mathrm{keV})$ is excluded due to a calibration problem.

\section{The Infrared and X-ray Correlation in the Oscillation State}

Study of the X-ray and infrared correlation of GRS 1915+105 can give us a direct insight of the disc-jet connection, since we can prove into the innermost part of the jet more freely from the optical depth effect than in the radio band. Most of previous infrared observations were conducted by small or middle-size telescopes given the relatively bright magnitude of GRS $1915+105(K \lesssim 13$ mag). In our campaign, however, we use the $8.2 \mathrm{~m}$ Subaru telescope to obtain infrared photometric light curves with unprecedented quality. This enables us to investigate the time variability of even 
very small amplitudes on a short time scale down to $1 \mathrm{sec}$ (for relevant works with the Palomar 5-m telescope refer to [19][20]|21].). Furthermore, thank to the campaign, almost continuous coverage in the X-ray band has been achieved by multiple satellites (Suzaku, Swift, and INTEGRAL) in the period of the Subaru observations.

Figure 6 shows simultaneous infrared and X-ray light curves taken with Subaru/ CISCO and Suzakul XIS-1 during the oscillation (Class $\theta$ ). As noticed, very small infrared flares with amplitudes of $<0.1$ mag are observed. There is anti-correlation between the infrared and X-ray fluxes; an infrared flare begins just after an X-ray soft dip. Analyzing all the simultaneous infrared and X-ray light curves, we sometimes find time delay of 0-3 minutes for the onset of an infrared flare measured from the beginning of an X-ray dip. Significant infrared variability is observed on a timescale of several seconds. This constrains the size of the infrared flaring region to be $\lesssim 10^{11} \mathrm{~cm}$.

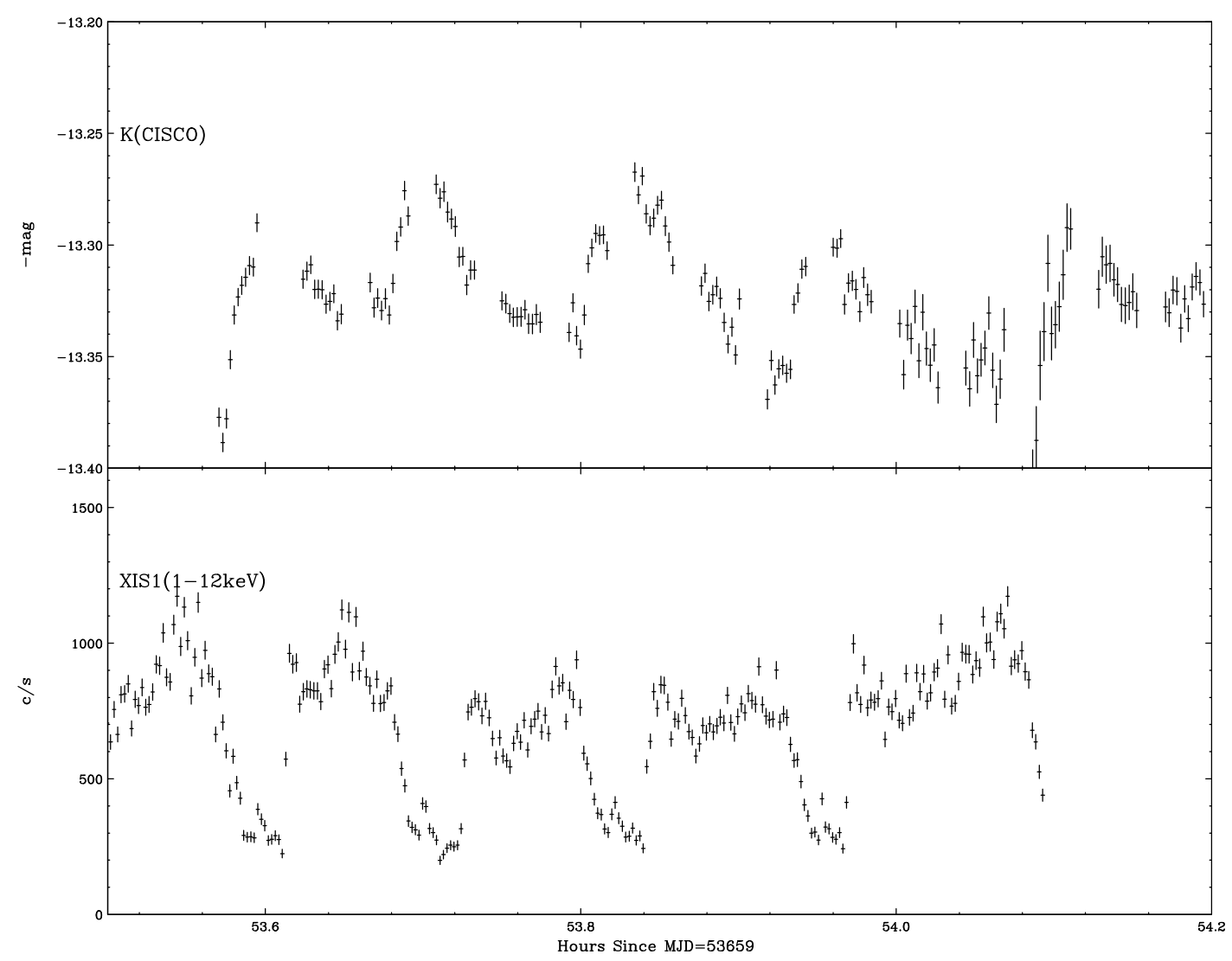

Figure 6: The simultaneous $K^{\prime}$ (upper) and X-ray (lower) light curves obtained with the Subaru/CISCO and Suzaku/XIS-1, respectively. The time resolution is $8 \mathrm{sec}$. Only statistical errors are indicated. In the Subaru light curve, a series of 12 data points corresponds to a single dithering position (see $\S 2.3$ ). We estimate that the relative systematic error between different positions could be 0.03 mag at maximum, but is negligible compared with the statistical error within a single position.

These results could be explained by an internal shock scenario discussed in [4], consistently with the model by [22]. The disc transition from State C (hard spectrum) to State A (soft) triggers the ejection of jet itself. This is now clearly demonstrated for the smallest class of jet in GRS 
1915+105 we observed. The same physics should hold for larger classes of jet as well (e.g., class $A$ and class $B$ jet in [20]). The ejected material will eventually flare-up by shock with preexisting matter, probably a slow jet accompanying the State $\mathrm{C}$ disc. The shock process can cause significant time delay between ejection and flare as observed; a flare can occur far away from the black hole at a distance of $\sim 10^{13} \mathrm{~cm}$. As discussed in [23], the amplitude of a flare depends on the efficiency of energy dissipation in the shock. In particular, the first ejection event after a long period of State C, such as "plateau" (for the class A jet) or "hard-dip" in Class $\beta$ [24] (for the class B jet), has the highest efficiency due to the presence of more material in the jet path, thus producing large infrared/radio flares.

\section{Summary}

We summarize the main conclusions from the 2005 October multiwavelength campaign of GRS 1915+105, focusing on the Suzaku and Subaru results.

1. The Suzaku spectra show that the iron-K feature changes with X-ray state. The disappearance of a narrow iron-K emission line in the oscillation high state suggests that the inner part of the disc becomes geometrically thick in this accretion mode, which shields the Comptonizing plasma as seen from the outer disc. The scale height of a disc wind increases in the oscillation state.

2. The Subaru observations establish that the disc transition from State $\mathrm{C}$ to State A triggers the ejection of jet. This causes a infrared flare taking place in a small region of $\lesssim 10^{11} \mathrm{~cm}$ at a distance of $0 \sim 10^{13} \mathrm{~cm}$ from the black hole, consistent with the internal shock scenario.

\section{Acknowledgement}

We thank Hiroshi Karouji, Tetsuo Nishimura, and Hajime Inoue for their supports and efforts in coordinating the simultaneous Subaru observations, Hisashi Hirabayashi and Bob Sault for the arrangement of the ATCA observations, Erik Kuulkers for the scheduling work of INTEGRAL, Tetsuya Nagata, Takahiro Kawadu, Hiroshi Hatano, and Takahiro Nagayama for the observations at the Infrared Survey Facility, Osamu Hashimoto and Eiji Nishihara for their supports at the Gunma Astronomical Observatory, and the Suzaku science working group members, including Tadayasu Dotani, Hiroshi Murakami, and Kiyoshi Hayashida, who helped us with the command operation of the Suzaku XIS. RATAN monitoring was supported by Russian Foundation Base Research (RFBR) grant N 05-02-17556 and mutual RFBR and Japan Society for the Promotion of Science (JSPS) grant N 05-02-19710. 


\section{References}

[1] Mirabel, I.F. \& Rodríguez, L.F. 1999, ARAA, 37, 409

[2] Fender, R.P. \& Belloni, T.M., 2004, ARAA, 42, 317

[3] Mitsuda, K., et al. 2006, PASJ, in press

[4] Ueda, Y. et al, 2002, ApJ, 571, 918

[5] Koyama, K., et al. 2006, PASJ in press

[6] Takahashi, T. et al. 2006, PASJ in press

[7] Motohara et al. 2002, PASJ, 54, 315

[8] Fender, R.P., Pooley, G.G., Brocksopp, G., \& Newell S.J. 1997, MNRAS, 290, L65

[9] Zerbi, F. et al., 2001, AN, 322, 275

[10] Tokunaga, A.T. \& Vacca, W.D., 2005, PASP, 117, 421

[11] Bertin, E. \& Arnouts, S. 1996, A\&AS 117, 393

[12] Stetson, P.B., 1987, PASP 99, 191

[13] Belloni, T., et al. 2000, A\&A, 355, 271

[14] Foster, R.S. et al. 1996, ApJ, 467, L81

[15] Mitsuda K., et al. 1984, PASJ, 36, 7

[16] Lee, J.C., et al. 2002, ApJ, 567, 1102

[17] Ueda, Y., et al. 2004, ApJ, 609, 325

[18] Kubota, A. et al, 2006, PASJ, in press (astro-ph/0610496)

[19] Eikenberry, S.S., et al. 1998, ApJ, 494, L61

[20] Eikenberry, S.S. et al. 2000, ApJ, 532, L33

[21] Rothstein, D.M., Eikenberry, S.S., \& Matthews, K. 2005, ApJ, 626, 991

[22] Fender R. P., Belloni, T. M., \& Gallo E. 2004, MNRAS, 355, 1105

[23] Vadawale, S.V. et al. 2003, ApJ, 597, 1023

[24] Mirabel, I.F. et al. 1998, A\&A, 330, L9 